Abstract

\title{
Cross Recurrence Quantification Analysis as a Tool for Detecting Rotors in Atrial Fibrillation: An In Silico Study ${ }^{\dagger}$
}

\author{
Andres Felipe Orozco ${ }^{1}$, Juan Pablo Ugarte ${ }^{2}$ and Catalina Tobón ${ }^{3}$ \\ 1 Universidad de Medellín, Medellín, Colombia \\ 2 Grupo de Investigación en Modelamiento y Simulación Computacional (GIMSC), Universidad de San \\ Buenaventura, Medellín, Colombia \\ 3 MATBIOM, Universidad de Medellín, Medellín, Colombia \\ + Presented at the Entropy 2021: The Scientific Tool of the 21st Century, 5-7 May 2021; Available online: \\ https://sciforum.net/conference/Entropy2021/.
}

Published: 5 May 2021

Atrial fibrillation is a cardiac arrhythmia characterized by rapid and irregular heartbeats that could be sustained by repetitive and cyclic activations around a core, known as rotors. Intracardiac electrograms studies have related the occurrence of a signal waveform pattern, called complex fragmented atrial electrograms (CFAE), with the surroundings of the rotor core.

Recurrence quantification analysis (RQA) has been proposed as a tool to detect CFAE. In RQA, the phase space trajectories are computed from the signal and the appropriates time delay embedding. In this work, we propose the computation of cross RQA (cRQA) using two distinct electrograms. We hypothesized that there is low recurrence rate when cRQA is estimated from a signal recorded near a rotor core and another in an adjacent point around the core.

We test the sample entropy, RQA and cRQA in five 2D in-silico simulations of atrial fibrillation sustained by different mechanisms: (i) a single stable rotor, (ii) a figure-of-eight re-entry with two stable rotors, (iii) a figure-of-eight re-entry with two meandering rotors, (iv) a single stable rotor and multiple propagating waves, (v) semi-stable rotors, meandering rotors and multiple propagating waves. Unipolar electrograms are simulated for each fibrillatory episode.

Our results show that, by applying sample entropy, the core of stable rotors, meandering rotors and multiples waves collision are associated with, by applying RQA the core of stable rotors and some areas with multiples waves collision exhibit low recurrence rates, notwithstanding meandering rotors were not detected. Differently, by applying cRQA only the cores of stable rotors are detected through low recurrence rates. These results suggest that CQRA could be a useful tool for stable rotors detection with high specificity. Future studies should include real electrograms recorded from patients with atrial fibrillation.

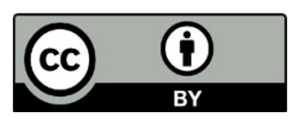

(C) 2021 by the authors. Licensee MDPI, Basel, Switzerland. This article is an open access article distributed under the terms and conditions of the Creative Commons Attribution (CC BY) license (http://creativecommons.org/licenses/by/4.0/). 